\title{
Reduced cluster search ML decoding for QO-STBC M-QAM MIMO systems
}

\section{Taufik Abrão* and Isaque Suzuki}

Electrical Engineering Department, State University of Londrina (DEEL-UEL), Rod. Celso Garcia Cid - PR445, s/n, Campus Universitário, P.O. Box 6001, Londrina, PR 86051-970, Brazil

Fax: +55-43-3371-4790

E-mail: taufik@uel.br

E-mail: isaquesuzuki@gmail.com

*Corresponding author

\section{Bruno A. Angélico}

Department of Electrical Engineering, Federal Technological University of Paraná (UTFPR), Av. Alberto Carazzai, 1640, Cornélio Procópio, PR 86300-000, Brazil Fax: +55-43-3520-4010

E-mail: bangelico@utfpr.edu.br

\section{Fernando Ciriaco and Paul Jean E. Jeszensky}

Laboratório de Comunicações e Sinais,

Department of Telecommunications and Control Engineering, Escola Politécnica of University of São Paulo (LCS-PTC-EPUSP),

Av. Prof. Luciano Gualberto, tr. 3, 158,

Cidade Universitária, São Paulo, SP,

S ao Paulo 05508-900, Brazil

Fax: +55-11-3091-5128

Fax: $+55-11-3091-5718$

E-mail: fciriaco@lcs.poli.usp.br

E-mail: pjj@lcs.poli.usp.br

\section{Fernando Casadevall Palacio}

Department of Signal Theory and Communication (TSC),

Universitat Politécnica de Catalunya (UPC),

Campus Nord, C/Jordi Girona, 1-3,

Barcelona 08034, Spain

Fax: 93-401-7424

E-mail: ferranc@tsc.upc.edu 
Abstract: Since the maximum likelihood (ML) decoding for quasi-orthogonal space-time block codes (QO-STBC) with inner and outer codes multipleinputmultiple- output (MIMO) antenna systems results too complex when the modulation order index and the number of receive antennas increases, an efficient reduced complexity ML-based decoding scheme is proposed in this contribution, aiming to reach promising QO-STBC coded MIMO system throughput $\times$ complexity trade-off. Under high-order modulation indexes $(16 \leq M)$-QAM, $4 \times n_{R}$ antennas, with $n_{R} \geq 1$, this work proposes a reduced cluster search ML decoder (RCS-ML) and compares the performancecomplexity with the ML decoding approach. Numerical results have indicated no degradation in performance and an increasing reduction in the complexity of RCS-ML decoder when the modulation order increases, been $12.5 \%$ of ML decoding complexity for $16-\mathrm{QAM}$, and $<1 \%$ for $256-\mathrm{QAM}$.

Keywords: MIMO system; quasi-orthogonal space-time block codes; QO-STBC; reduced complexity ML decoding; cluster search; space-time coding; STC.

Reference to this paper should be made as follows: Abrão, T., Suzuki, I., Angélico, B.A., Ciriaco, F., Jeszensky, P.J.E. and Casadevall Palacio, F. (2011) 'Reduced cluster search ML decoding for QO-STBC M-QAM MIMO systems', Int. J. Satellite Communications Policy and Management, Vol. 1, No. 1, pp.15-30.

Biographical notes: Taufik Abrão received his $\mathrm{BS}, \mathrm{MSc}$ and $\mathrm{PhD}$, all in Electrical Engineering from EPUSP - Escola Politécnica of University São Paulo, Brazil, in 1992, 1996, and 2001, respectively. He is currently an Associate Professor at the Electrical Engineering Department of UEL - State University of Londrina, Brazil. From 2007 to 2008, he was a Visiting Professor at TSC/UPC - Department of Signal Theory and Communications, Universitat Politécnica de Catalunya, Barcelona, Spain. His research interests include multi-user detection, MC-CDMA and MIMO systems, heuristic and optimisation aspects of multiple access systems and networking. He is the author or co-author of more than 70 research papers published in specialised periodicals and key conferences in the area of wireless communication and networking.

Isaque Suzuki received his BS and MSc in Electrical Engineering from State University of Londrina, Brazil in 2004 and 2009, respectively. He is currently working at Attack do Brasil Developing Digital Audio Processors and his research interests include signal processing, MIMO and STBC.

Bruno A. Angélico received his BS in Electrical Engineering from State University of Londrina in 2003 and MS in Electrical Engineering from Escola Politécnica of the University of São Paulo (EPUSP) in 2005. He was a Visiting Scholar at the California Institute for Telecommunications and Information Technology (Calit2), University of California, San Diego in 2007-2008. He is currently a PhD student at EPUSP, and an Assistant Professor at Federal Technological University of Paraná (UTFPR), Cornélio Procópio, Brazil. His research interests include signal processing, ultra wideband, and spread spectrum.

Fernando Ciriaco received his BS and MSc in Electrical Engineering from UEL - Londrina State University, Brazil in 2004 and 2006, respectively. He is currently a PhD student at EPUSP - Escola Politécnica of University of São Paulo, Brazil, a Coordinator and Professor at the Electrical Engineering Department of Faculdade Pitágoras, Brazil and also acts as a Coordinator of the 


\begin{abstract}
Wireless Biosignal Development Systems Group in GELT Tecnologia, Brazil, sponsored by FINEP. His research interests are multi-user detection, heuristic algorithms, MIMO, MC-CDMA systems, wireless communications, wireless biosignals and signal processing.

Paul Jean E. Jeszensky received his BS, MS and $\mathrm{PhD}$, all in Electrical Engineering from EPUSP-Escola Politécnica of University of São Paulo, Brazil, in 1972, 1981, and 1989, respectively. Since 1990, he has been with EPUSP where he is a Full Professor and Researcher in Communication Systems. He was a Visiting Professor at UPCUniversitat Politécnica de Catalunya, Barcelona, Spain, in 1995 and at TUB-Technical University of Budapest, Hungary, in 2001. He is the author of the book Sistemas Telefônicos (in Portuguese), Editora Manole, 2003 and his current research interests include CDMA systems, multi-user detection, code sequences analysis and related topics.
\end{abstract}

Fernando Jose Casadevall Palacio received his Engineer of Telecommunication and PhD Engineering from the Universitat Politécnica de Catalunya (UPC), Spain, in 1977 and 1983, respectively. He is currently a Full Professor in the Signal Theory and Communications Department. In the last ten years, he has mainly been concerned with the performance analysis and development of digital mobile radio systems. In particular, his research interests include cellular and personal communication system, multipath transceiver design, mobility and radio resources management, end to end QoS issues. During the last ten years, he participated in more than 20 research-projects founded by both public and private organisations.

\title{
1 Introduction
}

The last five years have been dominated by high demands on video and audio data with reliability real-time applications. In order to fulfill the requirements of high capacity systems, multiple-input-multiple-output (MIMO) schemes associated with space-time block codes (STBC) are frequently incorporated by many standards like WiMAX, specifically Alamouti Rate 1 STBC (Alamouti, 1998) (R1 STBC). In order to overcome the limitation of two-antennas while preserving full rate, Jafarkhani (2001) proposed QOSTBC schemes with reducing orthogonality but increasing its complexity. In Tirkkonen (2001), QO-STBCs performance were improved by using constellation rotation in order to achieve full diversity.

Furthermore, higher throughput with acceptable performance $\times$ complexity trade-off have been achieved through the inclusion of a bit-mapped coded modulation (BMCM) structure. For instance, BMCM in conjunction with parallel short LDPC, quasiorthogonal STBC scheme (Jafarkhani, 2005), in conjunction with iterative soft parallel interference cancellation (PIC) detector is discussed in Pau et al. (2008). The aim is to achieve relative low complexity schemes, high throughput with good performance and low processing delay in overall processing of detection and decoding. Good STBC designs must take into account the jointly optimisation of the following attributes: coding, diversity and multiplexing gains, and the decoder complexity as well.

Recently, the so called fast-decodable STBC MIMO schemes have been introduced aiming to reduce complexity with no degradation in performance. Previous works on 
fast-decodable low-complexity SBTC MIMO systems includes Biglieri et al. (2008), Jiang et al. (2008), Kim and Cheun (2008), Sezginer and Sari (2007), Choi et al. (2007) and Yuen et al. (2005). In Sezginer and Sari (2007), a family of full-rate, full-diversity $2 \times 2$ space-time coding (STC), and whose detection complexity grows only quadratically with the size of the signal constellation $(M)$ have been proposed. Thus, the optimum decoder complexity has been reduced by a factor of 256 for the 16-QAM signal constellation (and by 4,096 for the 64-QAM modulation).

A different approach to achieve low-complexity near-maximum likelihood (ML) QO-STBC decoding based on iterative interference cancellation (IICIS) was proposed in Kim and Cheun (2008). In the IICIS, the interference cancellation procedure (IC) was only used in the candidate vector pruning process and not in the detection process itself. Since the candidate pairs for decoding the transmitted symbol sub-vectors $s_{1}, s_{4}$ and $s_{2}, s_{3}$ in a QO-STBC scheme are updated at the end of two-consecutive IC stages, and simultaneously the number of candidate pairs decreases fast enough, few iterations are required to complete the decoding process. However, the overall decoder complexity still remains significant, especially for large modulation orders. Hence, aiming to reduce the overall decoding complexity, in Yuen et al. (2005), minimum decoding complexity (MDC) QO-STBC structures were proposed requiring the joint detection of two-real symbols regardless of the number of transmitting antennas.

Recently, an adaptive method selecting suitable MDC-QO-STBC codeword based on channel state information (CSI) has been proposed in Li et al. (2009). The CSI is used to calculate the inter-symbol interference (ISI) power of each optional MDC-QO-STBC. Then, the one with the minimum ISI power is the chosen codeword for the current transmission. For this adaptive method, it is possible to use linear decoding, achieving the lowest decoding complexity.

This contribution proposes a low-complexity efficient decoding algorithm for QO-STBCs schemes, based on reduced ML cluster search. The proposed RCS-ML decoder is suitable for any number of receive antennas, but the complexity advantage over ML decoding grows with the modulation order.

The remaining sections are divided as following. Section 2 describes the QO-STBC MIMO system. The proposed reduced cluster search ML decoding method in the context of QO-STBC codes is discussed in Section 4. Numerical results and complexity analysis for the proposed RCS-ML QO-STBC decoder with an arbitrary number of receive antennas are analysed in Section 5. Main conclusions are offered in Section 6.

\section{QO-STBC system model}

Let us consider a MIMO system with $n_{T}=4$ transmit antennas and $n_{R} \geq 1$ receive antennas, where four-symbols are transmitted simultaneously; additionally, $n_{T} n_{R}$ independent subchannels are assumed. Figure 1 illustrates the main characteristics of the adopted MIMO system. In this work, non-selective fading channels, $M$-QAM modulation, Rate 1 QO-STBC scheme of Jafarkhani (2005) were employed.

\subsection{Transmitter}

The baseband transmitter is constituted by a $M$-QAM modulator, where each QAM symbol is mapped using $m=\log _{2} M$ bits, a $1: n_{T}$ demultiplexer and a quasi-orthogonal STC 
block. In order to achieve high throughput (range of 2-8 bits per symbol period, depending on the modulation index), associated to suitable performance and simplicity of decoding at the receiver, the adopted modulation is high-order squared-QAM modulation with $m=2,4,6$ or 8 .

The QO-STBC transmitter topology employs Rate $1\left(R_{\mathrm{stbc}}=\frac{\text { \#symbols transmitted }}{\text { \#time slots used, } L}=1\right)$ quasi-orthogonal space-time block code (R1 QO-STBC) as proposed in Jafarkhani (2005), and described by the code matrix:

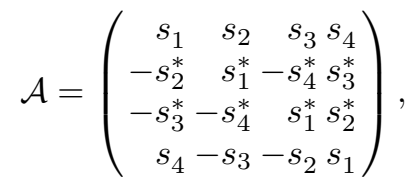

where $s_{i} \in \mathcal{S}$ is a $2^{m}$-ary constellation point, and $\mathcal{S}$ is a set of all valid QAM symbols belong to the adopted constellation at the transmitter. The corresponding overall throughput for a system using a $M$-ary constellation is defined by: $\Theta=R_{\text {stbc }} \log _{2} M=m$ [bits per symbol period].

Figure 1 QO-STBC MIMO system, suitable for high-order modulation $(M \geq 16)$ with reduced cluster search ML decoding approach (RCS-ML decoding)

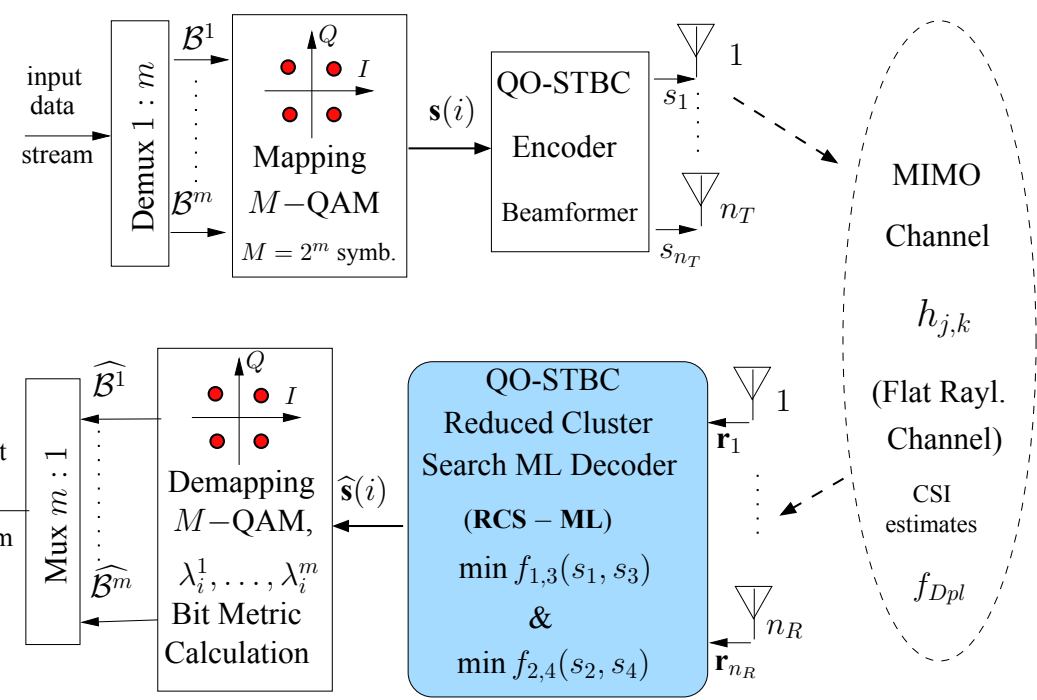

Note: $n_{T}$ transmitted and $n_{R}$ received antennas.

\subsection{Receiver}

Let $x(i)$ be the $i$ th symbol modulated with duration $T_{s}$, and $s_{j}(t)$ the transmitted symbol by the $j$ th transmit antenna at time $t$. Each transmitted symbol goes through the wireless channel to arrive at each of $n_{R}$ receive antennas. Denote the path gain from transmit antenna $j$ to receive antenna $k$ at each symbol interval by $h_{k j}(t)$. Hence, considering a baseband 
discrete-time model for a flat fading MIMO channel, the received signal at $k$ th antenna is given by:

$$
r_{k}(t)=\sum_{j=1}^{n_{T}} h_{k j}(t) s_{j}(t)+n_{k}(t), \quad t=1, \ldots, L
$$

where $L$ is the time slot size of STBC block, $h_{k j}(t), \forall k \in\left\{1,2, \ldots, n_{R}\right\}$, $\forall j \in\left\{1,2, \ldots, n_{T}\right\}$ are assumed to be either i.i.d. complex Gaussian random variables (fade amplitudes are Rayleigh distributed) with zero mean and $\mathbb{E}\left[\left(h_{k j}^{I}\right)^{2}\right]=\mathbb{E}\left[\left(h_{k j}^{Q}\right)^{2}\right]=\frac{1}{2}$, where $h_{k j}^{I}$ and $h_{k j}^{Q}$ are the real and imaginary parts of $h_{k j}(t)$, or the channel matrix is assumed unitary for the case of AWGN channel. The noise sample at the $k$ th receive antenna is assumed to be complex Gaussian with zero mean, and the samples $\left\{n_{k}\right\}, k=1, \ldots, n_{R}$ are admitted to be independents with variance:

$$
\mathbb{E}\left[n_{k}^{2}\right]=N_{0}=\frac{n_{T} \bar{E}_{s}}{\gamma}=\frac{n_{T} \bar{E}_{s}}{m 10^{\frac{\mathrm{sNR}}{10}} R_{\mathrm{stbc}}},
$$

where $\bar{E}_{s}$ is the average energy of the transmitted symbols, given a constellation format, $\gamma$ is the average received SNR per receive antenna (Jafarkhani, 2005), and SNR is the signal-to-noise ratio per receive antenna in decibels $(\mathrm{dB})$.

The received signals are sampled from all receive antennas, so (1) can be rearranged in a vectorial form, and for the $t$ th symbol period interval, the vector of the received signal is given by:

$$
\mathbf{r}(t)=\mathbf{H}(t) \mathbf{s}(t)+\mathbf{n}(t), \quad t=1, \ldots, L
$$

where for each time slot $t=1, \ldots, 4$ for the QO-STBC scheme,

$$
\mathbf{r}(t)=\left[r_{1}(t) r_{2}(t) \ldots r_{n_{R}}(t)\right]^{T}
$$

is the received signal vector, $\mathbf{s}(t)=\left[s_{1}(t) s_{2}(t) \ldots s_{n_{T}}(t)\right]^{T}$ is the transmitted symbol vector; the $n_{R} \times n_{T}$ channel matrix $\mathbf{H}(t)$, with channel coefficients $h_{k j}$ between the $j$ th transmitted antenna and $k$ th receive antenna, and $\mathbf{n}(t)=\left[n_{1}(t) n_{2}(t) \ldots n_{n_{R}}(t)\right]^{T}$ is the sampled noise vector. In a first analysis, the channel matrix coefficients are assumed to be known perfectly at the receiver, but completely unknown at the transmitter.

\subsection{ML decoding}

The ML decision metric is obtained by minimising the two-cost functions (Jafarkhani, 2001):

$$
\left(\widehat{s}_{1}, \widehat{s}_{2}, \widehat{s}_{3}, \widehat{s}_{4}\right)=\arg \min _{s_{1}, s_{4} \in \mathcal{S}} f_{1,4}\left(s_{1}, s_{4}\right), \arg \min _{s_{2}, s_{3} \in \mathcal{S}} f_{2,3}\left(s_{2}, s_{3}\right),
$$


where each cost function to be (independently) minimised are given by (5) and (6).

$$
\begin{aligned}
f_{1,4}\left(s_{1}, s_{4}\right) & =\sum_{k=1}^{n_{R}}\left[\left(\sum_{j=1}^{n_{T}=4}\left|h_{j k}\right|^{2}\right)\left(\left|s_{1}\right|^{2}+\left|s_{4}\right|^{2}\right)+2 \Re\left\{\left(-h_{1 k} r_{k}^{*}(1)\right.\right.\right. \\
& \left.-h_{2 k}^{*} r_{k}(2)-h_{3 k}^{*} r_{k}(3)-h_{4 k} r_{k}^{*}(4)\right) s_{1}+\left(-h_{4 k} r_{k}^{*}(1)\right. \\
& \left.+h_{3 k}^{*} r_{k}(2)+h_{2 k}^{*} r_{k}(3)-h_{1 k} r_{k}^{*}(4)\right) s_{4}+\left(h_{1 k} h_{4 k}^{*}\right. \\
& \left.\left.\left.-h_{2 k}^{*} h_{3 k}-h_{2 k} h_{3 k}^{*}+h_{1 k}^{*} h_{4 k}\right) s_{1} s_{4}^{*}\right\}\right]
\end{aligned}
$$

and

$$
\begin{aligned}
f_{2,3}\left(s_{2}, s_{3}\right) & =\sum_{k=1}^{n_{R}}\left[\left(\sum_{j=1}^{n_{T}=4}\left|h_{j k}\right|^{2}\right)\left(\left|s_{2}\right|^{2}+\left|s_{3}\right|^{2}\right)+2 \Re\left\{\left(-h_{2 k} r_{k}^{*}(1)\right.\right.\right. \\
& \left.+h_{1 k}^{*} r_{k}(2)-h_{4 k}^{*} r_{k}(3)+h_{3 k} r_{k}^{*}(4)\right) s_{2}+\left(-h_{3 k} r_{k}^{*}(1)\right. \\
& \left.-h_{4 k}^{*} r_{k}(2)+h_{1 k}^{*} r_{k}(3)-h_{2 k} r_{k}^{*}(4)\right) s_{3} \\
& \left.\left.+\left(h_{2 k} h_{3 k}^{*}-h_{1 k}^{*} h_{4 k}-h_{1 k} h_{4 k}^{*}+h_{2 k}^{*} h_{3 k}\right) s_{2} s_{3}^{*}\right\}\right]
\end{aligned}
$$

Note that the soft estimates symbols can be interpreted as $\widehat{\mathbf{s}}_{i}=\kappa \mathbf{s}_{i}+\eta_{i}$, where $\kappa$ is the coefficient of $\mathbf{s}_{i}$ in (5) or (6), and $\eta_{i}$ is the sum of two-terms at the receiver: additive white Gaussian noise (AWGN) plus interference term due to the quasi-orthogonality of the STBC scheme.

Hence, for MIMO system with small constellation size, it is computationally viable to evaluate, independently, all possible values for the pairs $\left(s_{1}, s_{4}\right)$ and $\left(s_{2}, s_{3}\right)$, using the two-cost functions (5) and (6), and directly obtain ML estimates. However, once the computation complexity increases exponentially with $m$, when the dimension of the constellation grows, i.e., $M \geq 16$, it is computationally inefficient to evaluate all of that pair combinations. Therefore, for high-order constellations, it becomes attractive to employ non-exhaustive procedures, or even sub-optimum methods in order to evaluate the QO-STBC cost functions. Section 3 identifies and illustrates the existence of certain pattern in (5) and (6), while Section 4 describes the proposed low complexity procedure to compute efficiently those cost functions. The procedure is specially suitable for high squared-order modulation MIMO QO-STBC with $n_{T}=4$ and any number of receive antennas.

\section{Pattern in cost function}

Basically, the ML detector consists in determining the point in a distorted constellation closer to the received symbol testing all valid symbol. Figure 2 shows three-distinct distorted 16-QAM constellations where point 2 is the closest point to the received symbol. 
Figure 2 Three typical cases of constellations distorted by the MIMO channel (modulo and phase of $h_{k j}$ ), darker stars

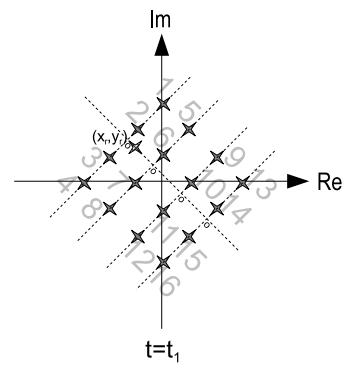

(a)

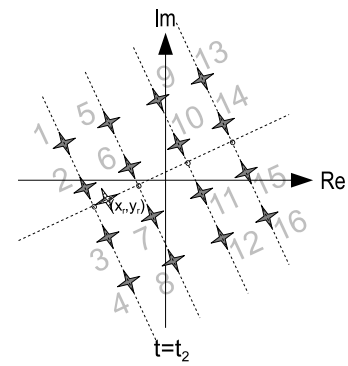

(b)

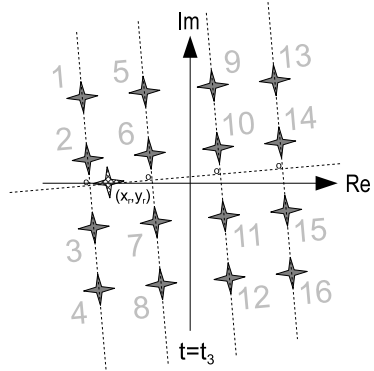

(c)

Note: Received symbol is the shadowed star, identified by the coordinates $\left(x_{r}, y_{r}\right)$.

In Figure 2(a), $t=t_{1}$ we have $|h|<1$ and $\angle h<0$, Figure 2(b) $t=t_{2}$ we have $|h|=1$ and $\angle h>0$ and Figure 2(c) $t=t_{3}$ we have $|h|>1$ and $\angle h<0$.

In order to identify patterns and clusters, we start with an analytical description to calculate the distances between received symbol and a valid symbol from the distorted constellation. Given a SISO system using a square M-QAM constellation and considering that the distance between constellation symbols is $A$, as shown in Figure 3, we can express the $\sqrt{M}$ vertical lines $r_{p}$ as:

$$
r_{p}: \quad x_{p}+0 \cdot y_{p}=\frac{-\left[\left(\log _{2} M-1\right)+2(p-1)\right]}{2} A, \quad p=1, \ldots, \sqrt{M} .
$$

Each received symbol affected by the channel is scaled by the factor $\|h\|$ and rotated by $\angle h$. But, to simplify the analysis, it will be applied the inverse effect caused by the channel to the received symbol; hence, the relative distances remain the same. In doing so, the received symbol $\left(\breve{x_{r}}, \breve{y_{r}}\right)$ can be written as:

$$
\begin{aligned}
\breve{x_{r}} & =\frac{1}{\|h\|}\left[\cos (-\angle h) x_{r}-\sin (-\angle h) y_{r}\right] \\
\breve{y_{r}} & =\frac{1}{\|h\|}\left[\sin (-\angle h) x_{r}+\cos (-\angle h) y_{r}\right]
\end{aligned}
$$

The distance between any point $\left(x_{p}, y_{p}\right)$, belongs to line $r_{p}$, and the received symbol $\left(\breve{x_{r}}, \breve{y_{r}}\right)$ is:

$$
d_{r, p}=\sqrt{\left(\breve{x_{r}}-x_{p}\right)^{2}+\left(\breve{y_{r}}-y_{p}\right)^{2}} .
$$

Substituting (7) in (10):

$$
\begin{aligned}
& d_{r, p}= \\
& \sqrt{\left(\breve{x_{r}}-\frac{-\left[\left(\log _{2} M-1\right)+2(p-1)\right]}{2} A\right)^{2}+\left(\breve{y_{r}}-y_{p}\right)^{2}},
\end{aligned}
$$


and replacing (8) and (9) in (11) and squaring both sides results:

$$
\begin{aligned}
d_{r, p}^{2} & =\left(\frac{1}{\|h\|}\left[\cos (-\angle h) x_{r}-\sin (-\angle h) y_{r}\right]-\right. \\
& \left.\frac{-\left[\left(\log _{2} M-1\right)+2(p-1)\right]}{2} A\right)^{2}+ \\
& +\left[\frac{1}{\|h\|}\left[\sin (-\angle h) x_{r}+\cos (-\angle h) y_{r}\right]-y_{p}\right]^{2} .
\end{aligned}
$$

Considering that $M, h$ and $A$ are constants during a symbol period, we ready obtain:

$$
\begin{aligned}
d_{r, p}^{2} & =C(p)+\left(K-y_{p}\right)^{2}, \quad \text { where } \\
K & =\frac{1}{\|h\|}\left[\sin (-\angle h) x_{r}+\cos (-\angle h) y_{r}\right] \\
C(p) & =\left(\frac{1}{\|h\|}\left[\cos (-\angle h) x_{r}-\sin (-\angle h) y_{r}\right]-\right. \\
& \left.\frac{-\left[\left(\log _{2} M-1\right)+2(p-1)\right]}{2} A\right)^{2}
\end{aligned}
$$

Figure 3 Two-square 16-QAM constellation mapping
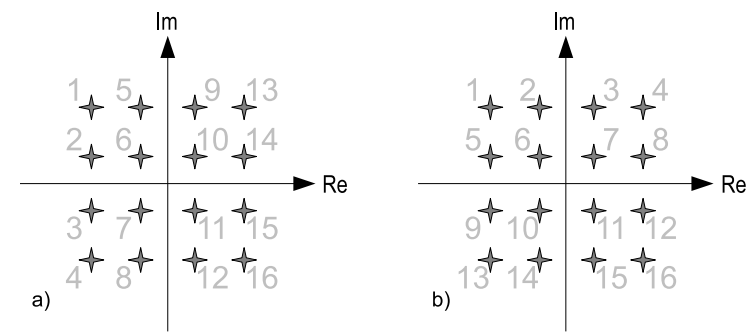

Analysing equation (15) we can see that it varies only in $p$ and $K$ is constant during a symbol period as we can see in (14). From (13), the distance $d_{r, p}$ is $\left(K-y_{p}\right)^{2}$ summed to $C(p)$, that is constant given $p$ fixed. Furthermore, observing Figure 3(b), the imaginary part of points $\{1,2,3,4\}$ are the same and this is true for the points $\{5,6,7,8\}$, and so on. The only difference between the distance from the received point to any line $r_{p}$ is the constant $C(p)$ pointed out in Figure 4(b). The component $\left(K-y_{p}\right)^{2}$ is the repeating pattern that appears in Figure 4.

So, repeating patterns will appear in the evaluation of the distance between received symbol and a square M-QAM constellation with equiprobable symbols. As a result, these pattern repeats itself at each $\sqrt{M}$ symbols. 
Figure 4 Patterns in cost function depending on the distance between symbol received and distorted constellation for 16-QAM SISO system

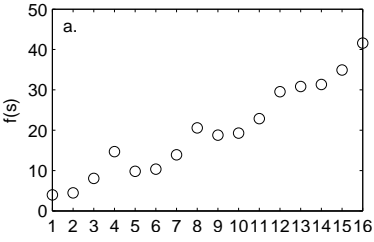

(a)

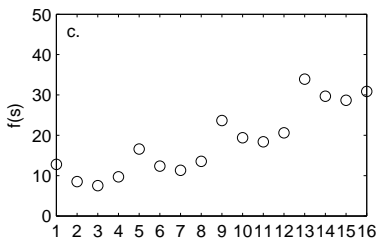

(c)

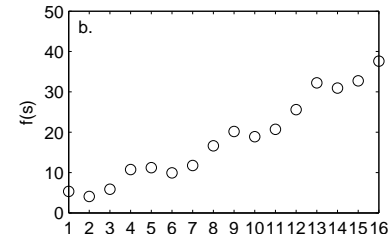

(b)

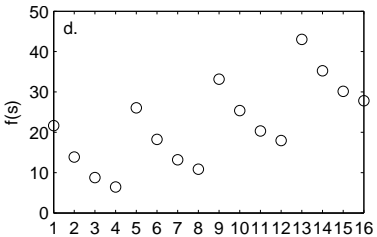

(d)

Notes: Received symbol closer to (a) constellation point 1, (b) constellation point 2 ,

(c) constellation point 3, (d) constellation point 4 .

\subsection{Extension to MIMO systems}

The optimum detector that minimises the average probability of error is the ML detector. It performs the following optimisation:

$$
\min _{\mathbf{s} \in \mathcal{S}}\|\mathbf{r}-\mathbf{H s}\|^{2}
$$

where $\mathcal{S}$ is the set of all possible combinations of $\left(s_{1}, s_{2}, \ldots, s_{n_{T}}\right)$ of a constellation $M$-QAM. Rewriting (16) we have that:

$$
\begin{aligned}
& \min _{\mathbf{s} \in \mathcal{S}}\|\mathbf{r}-\mathbf{H s}\|^{2} \\
& \min _{\mathbf{s} \in \mathcal{S}} \sum_{k=1}^{n_{R}}\left(r_{k}-\mathbf{h}_{k} \mathbf{s}\right)^{2} \\
& \min _{\mathbf{s} \in \mathcal{S}} \sum_{k=1}^{n_{R}}\left[r_{k}-\sum_{j=1}^{n_{T}} h_{k j} s_{k}\right]^{2} \\
& \min _{\mathbf{s} \in \mathcal{S}} \sum_{k=1}^{n_{R}}\left[r_{k}-\left(h_{k 1} s_{1}+h_{k 2} s_{2}+\ldots+h_{k n_{T}} s_{n_{T}}\right)\right]^{2}
\end{aligned}
$$

Considering equation (20) and that $n_{R}=1$, if we let $s_{1}$ varying and hold $s_{2}, \ldots, s_{n_{T}}$ fixed; hence, equation (20) describes the same problem of previous section (SISO system) i.e., the 
distance from a point to a set of lines. Then, we can conclude that patterns will appear at each $\sqrt{M}$ symbols. The same conclusion is valid if we vary $s_{2}$ and hold the other values variable fixed. So, to the MISO case there will be $n_{T}$-dimensional pattern on each hypercube of $\sqrt{M}$ symbols. In the MIMO case, where $n_{R}>1$, the total cost function is the sum of many cost functions that have patterns and it will also show patterns.

\section{Reduced cluster search ML decoding (RCS-ML)}

Our idea of cluster search arises after observing a pattern over cost functions behaviour, equations (5) and (6), considering all possible symbol combinations. As we can see from Figure $5(\mathrm{a})$, there are patterns that repeat itself. This figure was generated considering 16-QAM modulation; so $4 \times 4$ clusters can be identified.

Figure 5 Typical $f_{14}$ values, considering $\left(s_{1}, s_{4}\right)$ symbol pair in a 16-QAM modulation (a) for all $\left(s_{1}, s_{4}\right)$ values, (b) for only one cluster showing local minimum, (c) for all local minima, showing global minimum
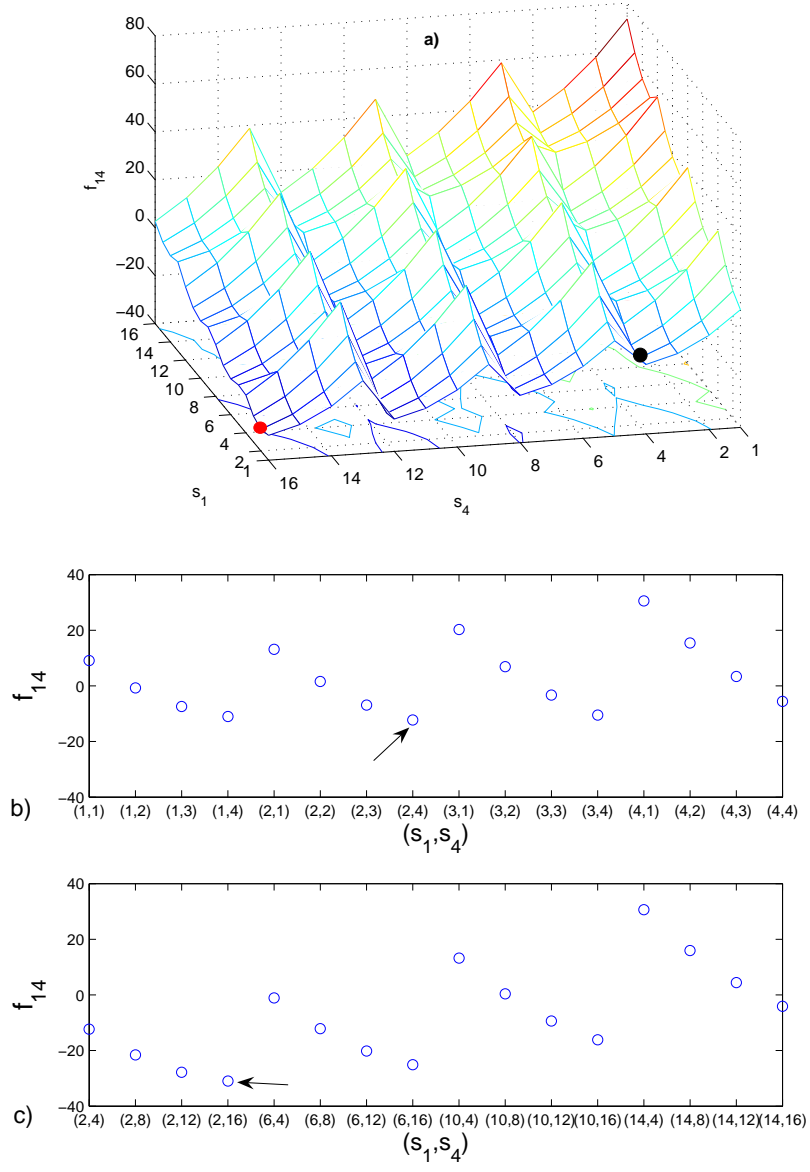
Figure 6 Example of RCS decoding and 16-QAM, (a) search space divided in clusters (b) first cluster to be explored (c) cost for each pair $\left(s_{1}, s_{4}\right)$ inside first cluster (d) local minima with respective cost value

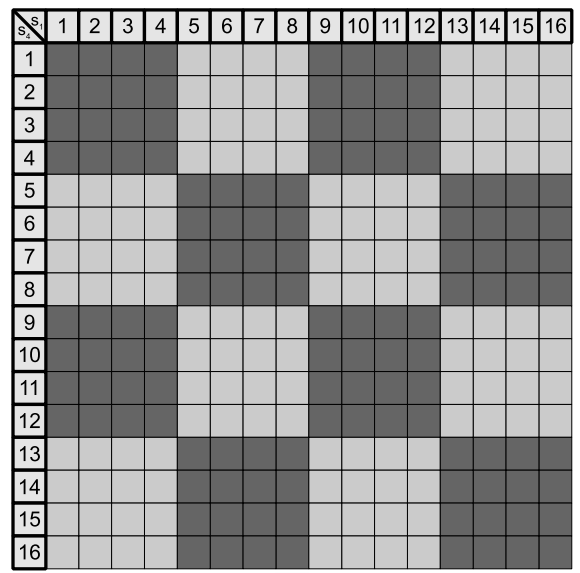

(a)

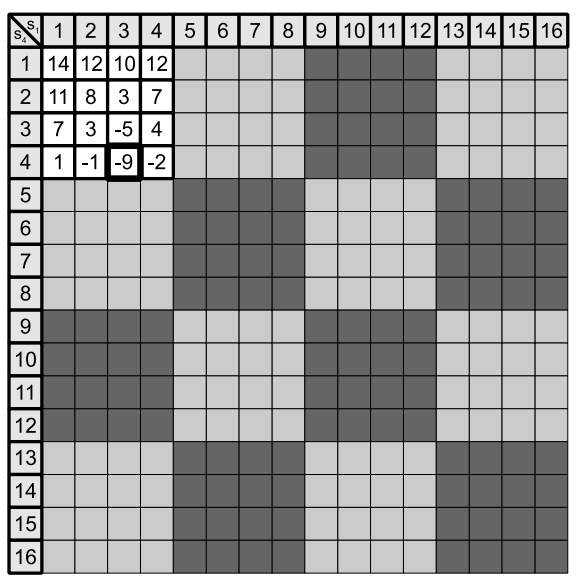

(c)

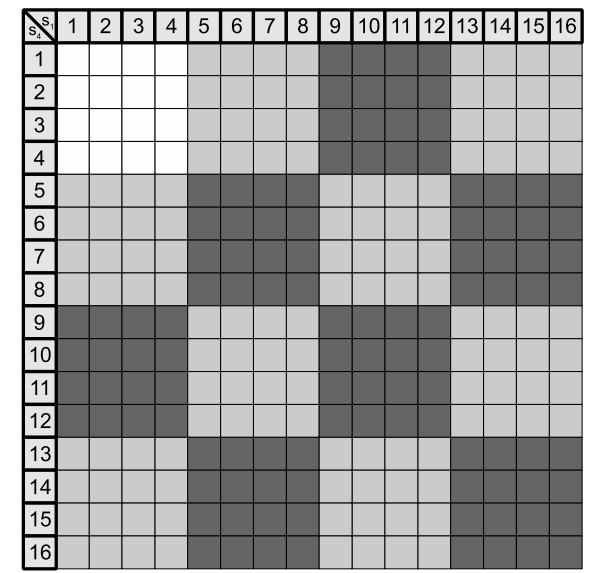

(b)

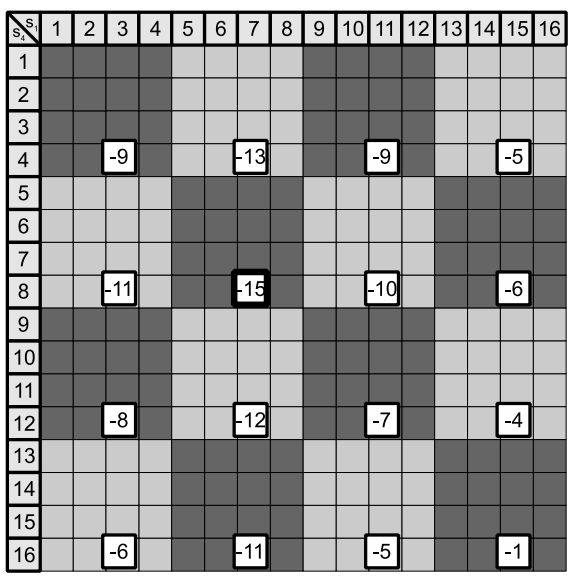

(d)

The idea is to perform an ML search only inside a cluster (we choose the first cluster in Figure 5(b), defining the relative coordinates of the pair that minimised (5) [or alternatively (6)] and generate a set with all pairs of symbols with the same coordinates inside the other clusters. This pattern repeats itself at each $\sqrt{2^{m}}$ symbols for symbol pairs $\left(s_{1}, s_{4}\right)$ [alternatively $\left(s_{2}, s_{3}\right)$ ], and all pairs inside this pattern constitutes a cluster. Finally, perform a ML search over the generated set and choose the one that minimises (5) and (6), Figure 5(c) shows the values for $f_{1,4}$ in the cluster which global minimum occurrence $\left(s_{4}=\underline{16}\right)$.

In order to implement the RCS-ML QO-STBC decoding, three-steps are carry out as described in Algorithm 1. These steps are valid to find ML solution for each symbol pair, $\left(s_{1}, s_{4}\right)$ or $\left(s_{2}, s_{3}\right)$ in the QO-STBC scheme. 
Algorithm 1 RCS-ML over $\left(s_{1}, s_{4}\right)$ symbol pair

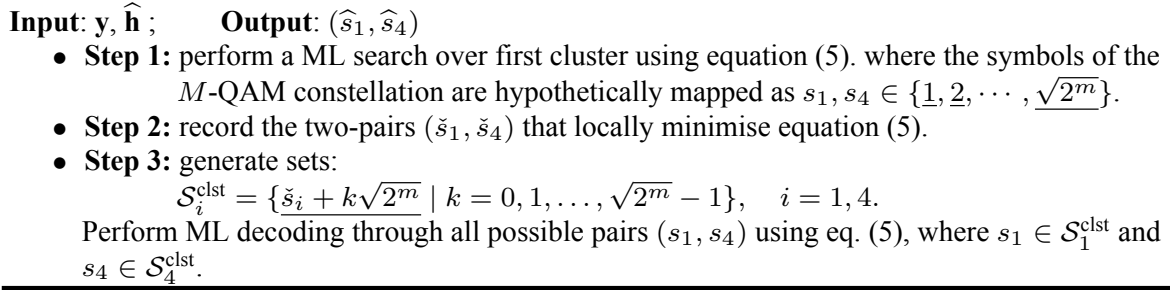

Figure 6 shows an example of RCS-ML procedure for 16-QAM modulation. In this case, the modulation order is $m=4$, and as $\sqrt{2^{m}}=4$, then, there are $4 \times 4$ clusters, Figure 6 (a). We can see from Figure 6(b). the first cluster in white color, which is composed by 16 pairs $\left(s_{1}, s_{4}\right)$, where $s_{1}, s_{4} \in\{\underline{1}, \underline{2}, \underline{3}, \underline{4}\}$. In Figure $6(\mathrm{c})$, a local minimum is found minimising (5), and in Figure 6(d). the global minimum is found from all clustered pairs selected in Step 3. In this case, the algorithm finds $\left(s_{1}, s_{4}\right)=(7,8)$. This process is repeated considering the other symbol pair $\left(s_{2}, s_{3}\right)$ and equation (6).

\section{Numerical results}

The main system and channel parameters used in Monte Carlo simulations are summarised in Table 1. In all numerical results shown here, QO-STBC scheme from Jafarkhani (2001) were adopted. For simplicity, in this section, only perfect know of CSI at the receiver side was assumed.

In accordance with the most common channel model in the literature (Jafarkhani, 2001; Alamouti, 1998), herein, we consider quasi-static time-varying channel model: the fading coefficients remain fixed during each QO-STBC block of $L=4$ time slots and vary independently from one-block to the next (quasi-static fading).

Table 1 MIMO system, RCS-ML decoding and channel parameters

\begin{tabular}{lc}
\hline Parameter & Adopted values \\
\hline QO-STBC MIMO system & $n_{T}=4$ \\
\# Tx antennas & $n_{R}=1$ and 4 \\
\# Rx antennas & Squared $M$-QAM: $M=4,16,64,256$ \\
Modulation format & Rate $1, R_{\text {stbc }}=1($ Jafarkhani, 2001) \\
QO-STBC code & $S N R[-2.5 ; 30] \mathrm{dB}$ \\
Received SNR per antenna & $\Theta=1.0,2.0,3.0,4.0[$ bits $/$ symb. period $]$ \\
Throughput & Flat-frequency \\
Rayleigh channel & Quasi-static (slow), $L=4$ \\
Sub-channel fading & Perfectly known at Rx \\
Channel type & \\
Channel state info. & $\sqrt{2^{m}} \times \sqrt{2^{m}}$ \\
RCS- $M L$ decoding & \\
Cluster size &
\end{tabular}

Figure 7 describes the Monte-Carlo simulation results for the case of QO-STBC MIMO system with $n_{T}=4 \times n_{R}=4$ antennas employing the ML against RCS-ML decoding; while Figure 8 compares the performance of both decoders but considering $n_{R}=1$ receive 
antenna. One can observes that the system performances with RCS-ML and conventional ML decoding are undistinguished for all SNR levels and constellation size conditions.

Figure 7 ML against RCS-ML decoding performance comparison for the QO-STBC MIMO system; $n_{T}=4 \times n_{R}=4$ antennas

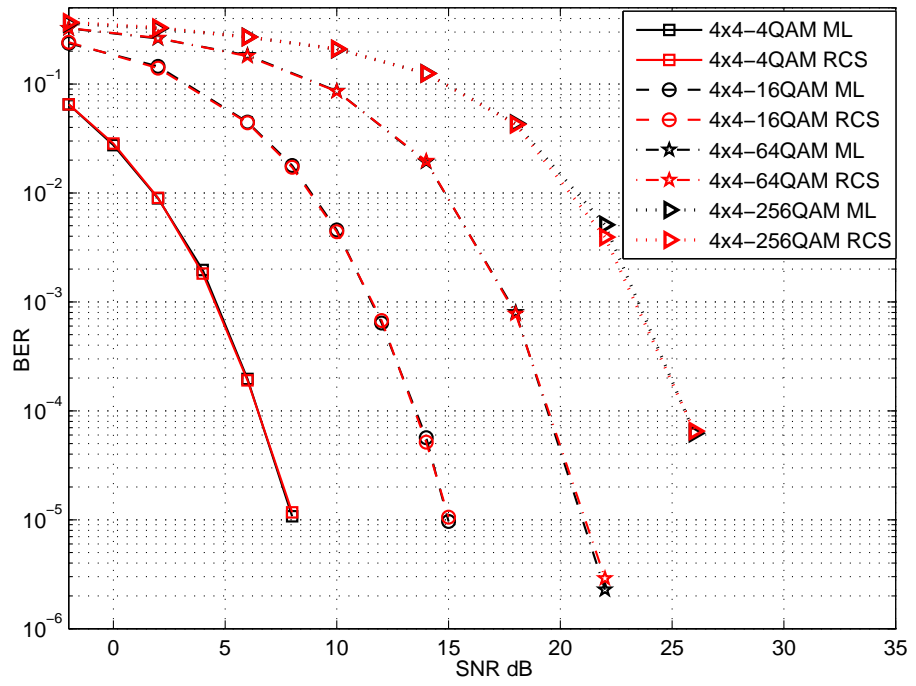

Figure 8 ML against RCS-ML decoding performance comparison for the QO-STBC MIMO system with different QAM constellation size, $n_{T}=4 \times n_{R}=1$ antennas

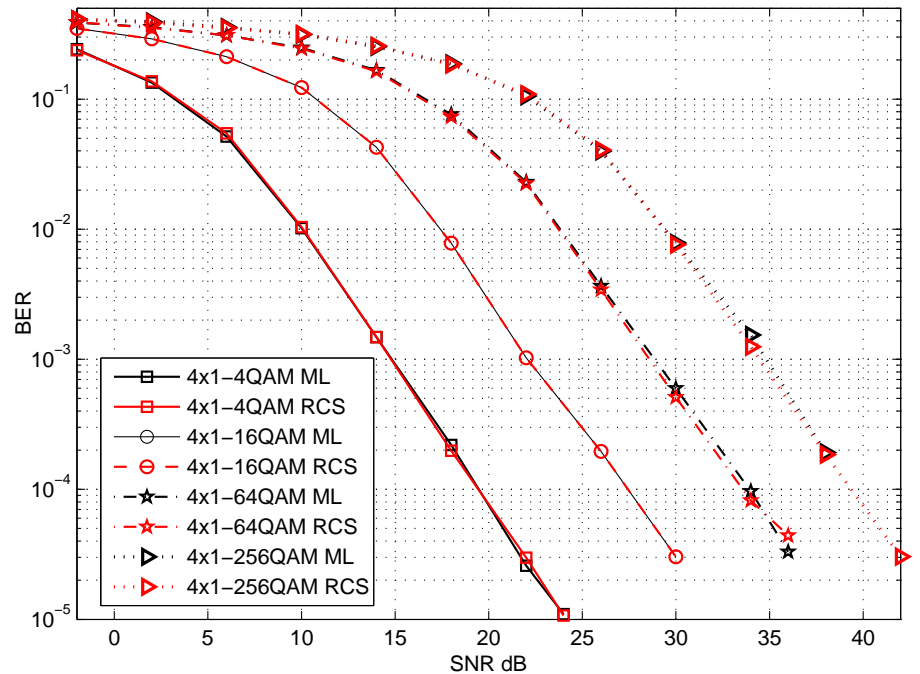

\subsection{Complexity analysis for the RCS-ML decoding}

In order to evaluate the algorithm complexity, we consider real multiplications and sums. Analysing (5) and (6) and taking into account each complex multiplication as four-real 
multiplications and each complex sum as two-real sums, we have 90 real multiplications and 27 real sums for each $f_{14}$ or $f_{23}$ evaluation. Once the RCS-ML algorithm needs only $2^{m}$ ML evaluation for Step 1 and $2^{m}$ for Step 2, so, the overall ML evaluation number is only $2^{m+1}$ evaluation to proceed the ML QO-STBC symbol decoding. Table 2 compares our RCS-ML QO-STBC decoding complexity with that necessary to proceed the ML QO-STBC decoding. Those complexities, in terms of real multiplications and sums, that are necessary to perform QO-STBC decoding, can be compared through the perceptual complexity reduction factor, expressed by

$$
\mathrm{CR}=\frac{\mathrm{C}_{R C S}}{\mathrm{C}_{M L}} \times 100 \quad[\%] .
$$

For all the analysed constellations and number of received antennas, this complexity reduction ratio is simply given by $\mathrm{CR}=2^{1-m} \times 100[\%]$.

Table 2 Number of real multiplications/sums per receive antenna per symbol pair necessary for QO-STBC decoding, $n_{T}=4 \times n_{R}=1$

\begin{tabular}{lcccc}
\hline Decoder & $4-Q A M$ & $16-Q A M$ & $64-Q A M$ & $256-Q A M$ \\
\hline $\mathrm{RCS}$ & $720 / 216$ & $2880 / 864$ & $11520 / 3456$ & $46080 / 13824$ \\
$\mathrm{ML}$ & $1440 / 432$ & $23040 / 6912$ & $368640 / 110592$ & $5898240 / 1769472$ \\
\hline $\mathrm{CR}_{\mathrm{RCS}}$ & $50 \%$ & $12.5 \%$ & $3.125 \%$ & $0.781 \%$ \\
\hline
\end{tabular}

\section{Conclusions}

In this contribution, we have proposed a reduced complexity ML decoding scheme based on cluster search, suitable for QO-STBC MIMO systems with higher-order modulation indexes and any number of receive antennas.

Thanks to reduced cluster search procedure, the numerical results for the proposed ML decoding have indicated no degradation in the performance, achieving the ML performance in all cases analysed, with a significative increasing reduction in the computational complexity when the modulation order increases, been $12.5 \%$ of ML decoding complexity for $16-\mathrm{QAM}$, and $<1 \%$ for $256-\mathrm{QAM}$.

\section{References}

Alamouti, S. (1998) 'A simple transmit diversity technique for wireless communications', IEEE Journal on Selected Areas in Communications, Vol. 16, No. 8, pp.1451-1458.

Biglieri, E., Hong, Y. and Viterbo, E. (2008) 'On fast-decodable space-time block codes', in IEEE International Zurich Seminar on Communications, pp.116-119.

Choi, D., Chae, C. and Jung, T. (2007) 'Design of new minimum decoding complexity quasi-orthogonal space-time block code for 8 transmit antennas', in IEEE International Symposium on Signal Processing and Information Technology, pp.674-677.

Jafarkhani, H. (2001) ‘A quasi-orthogonal space-time block code’, IEEE Transactions on Communications, Vol. 49, No. 1, pp.1-4. 
Jafarkhani, H. (2005) Space-Time Coding: Theory and Practice, Cambridge University Press.

Jiang, C., Zhang, H., Yuan, D. and Chen, H-H. (2008) 'A low complexity decoding scheme for quasiorthogonal space-time block coding', in SAM 2008 - 5th IEEE Sensor Array and Multichannel Signal Processing Workshop, pp.9-12.

Kim, J. and Cheun, K. (2008) 'An efficient decoding algorithm for QO-STBC based on iterative interference cancellation', IEEE Communications Letters, Vol. 12, No. 4, pp.292-294.

Li, W., Hua, J., Hui, Z. and Wenbo, W. (2009) 'Adaptive full-diversity full-rate space-time block code with linear decoding complexity', in IEEE International Symposium on Microwave, Antenna, Propagation and EMC Technologies for Wireless Communications, pp.1221-1225.

Pau, N.S.J., Taylor, D.P. and Martin, P.A. (2008) 'Robust high throughput space time block codes using parallel interference cancellation', IEEE Transactions on Wireless Communications, Vol. 7, No. 5, pp.1603-1613.

Sezginer, S. and Sari, H. (2007) 'Full-rate full-diversity $2 \times 2$ space-time codes of reduced decoder complexity', IEEE Communications Letters, Vol. 11, No. 12, pp.973-975.

Tirkkonen, O. (2001) 'Optimizing space-time block codes by constellation rotations', in Proc. Finnish Wireless Communications Workshop,pp.59-60.

Yuen, C., Guan, Y.L. and Tjhung, T.T. (2005) 'Quasiorthogonal stbc with minimum decoding complexity: further results', in IEEE Wireless Communications and Networking Conference, Vol. 1, pp.483-488. 